\title{
Identification of genes, associated with black pigmentation of seeds in cereals, based on transcriptomic analysis
}

\author{
A.Yu. Glagoleva ${ }^{1,2 *}$, N.A. Shmakov², O.Yu. Shoeva², G.V. Vasiliev ${ }^{2}$, \\ N.V. Shatskaya ${ }^{2}$, A. Börner ${ }^{3}$, D.A. Afonnikov ${ }^{1,2}$, E.K. Khlestkina ${ }^{1,2,4}$ \\ ${ }^{1}$ Novosibirsk State University, Novosibirsk, Russia \\ ${ }^{2}$ Institute of Cytology and Genetics SB RAS, Novosibirsk, Russia \\ ${ }^{3}$ Leibniz Institute of Plant Genetics and Crop Plant Research (IPK), Gatersleben, Germany \\ ${ }^{4}$ N.I. Vavilov All-Russian Research Institute of Plant Genetic Resources (VIR), Saint-Petersburg, Russia \\ *e-mail: glagoleva@bionet.nsc.ru
}

Key words: barley, near-isogenic lines, phytomelanin, Blp, RNA-seq, differetial expression

Motivation and Aim: Some plant species have 'melanin-like' black seed pigmentation. However, the chemical and genetic nature of this 'melanin-like' black pigment has not yet been fully explored due to its complex structure and ability to withstand almost all solvents. Nevertheless, identification of genetic networks participating in trait formation is key to understanding metabolic processes involved in the expression of 'melaninlike' black seed pigmentation. The aim of the current study was to identify differentially expressed genes (DEGs) in barley near-isogenic lines (NILs) differing by allelic state of the Blp (black lemma and pericarp) locus.

Methods and Algorithms: We used FastQC to estimate sequencing quality, cutadapt to remove adapter sequences and prinseq to filter sequences by quality and length. Filtered libraries were mapped to $\mathrm{H}$. vulgare genome assembly version $32,608 \mathrm{v} 1.33$ from the Ensembl Plants database (http://plants.ensembl.org). Mapping was performed with TopHat 2 tool. The resulting alignments were processed with the Cufflinks v 2.2.1 pipeline. Pathway analysis was performed using PlantCyc database (http:/www. plantcyc.org/). After that selected DEGs were verified using qRT-PCR.

Results: Firstly, we excluded involvement of metabolic pathways, known for other types of pigments (i.e. widespread flavonoid pigments). Our data demonstrated that none of the key flavonoid biosynthesis genes is expressed significantly in black-grained barley during the pigment formation. Then, we used RNAseq approach to reveal DEGs in black-colored and uncolored-grained barley. A total of 957 genome fragments had statistically significant changes in expression levels between lines BLP and BW, with 632 fragments having increased expression levels in line BLP and 325 genome fragments having decreased expression. Genes with high level of differential expression in BLP line were identified in following pathways: suberin monomer biosythesis, diterpene phytoalexins precursors biosynthesis, cutin biosynthesis, cutular wax biosynthesis, and phenylpropanoid biosynthesis, initial reactions.

Conclusion: The number and diversity of metabolic pathways confirms our earlier assumption about the pleiotropic nature of the Blp locus. Besides forming a black color of the seeds, Blp locus is associated with the resistance to oxidative stress (possibly due to the increased content of antioxidants, including ferulic acid), as well as increased resistance to pathogens (due to the synthesis of phytoalexins and cuticular waxes). The black color of the seeds is presumably associated with the action of the enzyme polyphenol oxidase (PPO). Acknowledgements: The study was supported by the Russian Science Foundation (No. 16-14-00086). 